\title{
Efficacy and safety of insulin glulisine in the treatment of gestational diabetes
}

\begin{abstract}
Aim: To date, only two rapid-acting insulin analogs have been shown to be safe and effective for the treatment of diabetes during pregnancy: insulin aspart and insulin lispro. Our objective was to evaluate the safety and efficacy of insulin glulisine for the treatment of postprandial hyperglycemia in women with gestational diabetes (GDM).
\end{abstract}

Methods: Within a single-center randomized controlled trial, we evaluated 16 women with GDM in two parallel arms. All women were on basal treatment with insulin NPH; 6 women randomized to the study arm (insulin glulisine) and 10 were in the control arm utilizing the current standard of care for meal-related bolus insulin (insulin lispro). In addition to weekly study visits, women participated in a four-hour in-clinic meal challenge which involved a standardize meal and blood glucose (YSI) measurements. Statistical analysis was based on women who participated in the meal challenge and had more than 2 weekly visits ( 3 study arm women and 8 control arm women). Participants were all Latina and were between 24 and 38 years old

Results: At enrollment, mean gestational age was 30.3 weeks with mean hemoglobin A1C (HbA1c) 6.0 in the study arm and 27.9weeks with mean HbA1c 5.7 in the control arm. Throughout the study, the average postprandial glucose (PPG) and HbA1c measurements were $113 \mathrm{mg} / \mathrm{dL}$ and 6.0 , respectively, for the study arm and $109 \mathrm{mg} / \mathrm{dL}$ and 5.6, respectively, for the control arm; these did not significantly differ between the two study groups ( $\mathrm{p}$-value $=0.5$ for PPG comparison and $\mathrm{p}$-value $=0.06$ for HbA1c comparison). Regarding the meal challenge, an equal number of women had peak blood glucose at 75 minutes after the meal $(\mathrm{N}=2$ study arm and $\mathrm{N}=2$ control arm).

Conclusion: Results of this pilot study suggest that using insulin glulisine for the treatment of postprandial hyperglycemia in pregnant women with GDM may be safe and effective, but should be further evaluated in larger clinical trials.

Keywords: gestational diabetes mellitus, insulin glulisine, hyperglycemia
Volume 9 Issue 2 - 2018

\author{
Kristin Castorino DO, Maggi Beckstrand, \\ Ceara Axelrod \\ William Sansum Diabetes Center, USA
}

Correspondence: Kristin Castorino DO,William Sansum Diabetes Center, 2219 Bath Street, Santa Barbara, CA 93105, Tel (805) 682-7640, Fax (805) 682-3332, Email kcastorino@sansum.org

Received: February 27, 2018 | Published: April 30, 2018

\section{Abbreviations}

GDM, gestational diabetes mellitus; PPG, postprandial glucose; SBCPHD-DPC, santa barbara county public health department diabetes in pregnancy clinic, LGA, large for gestational age; PPG, postprandial glucose; $\mathrm{HbA} 1 \mathrm{c}$, hemoglobin A1C

\section{Introduction}

In $2015,33.9 \%$ or approximately 84.1 million U.S. adults aged 18 years or older had prediabetes. ${ }^{1}$ Gestational diabetes is akin to a pre-diabetic state, and affects both maternal and fetal morbidity and mortality. ${ }^{2}$ Gestational diabetes affects $2 \%$ to $10 \%$ of pregnancies. ${ }^{2}$ In the post-partum period, $5 \%$ to $10 \%$ of women with gestational diabetes are found to have diabetes, usually type 2 . Among those women who have had gestational diabetes not determined to be type 1 or 2 postpartum, there is a $35 \%$ to $60 \%$ chance of developing type 2 diabetes in the next 10-20 years. ${ }^{3}$ Unfortunately, the prevalence of GDM can be as high as $14 \%$ in high risk groups, especially in minority and socioeconomically disadvantaged populations. ${ }^{3}$

New diagnostic criteria for gestational diabetes have been adopted by multiple organizations such as the American Diabetes Association and the International Association for Diabetes in Pregnancy Study Group. This new one-step $75 \mathrm{gram}$ oral glucose tolerance diagnostic test would increase the proportion of women diagnosed with gestational diabetes. Using these new diagnostic criteria, an international, multicenter study of gestational diabetes found that $18 \%$ of the pregnancies were affected by gestational diabetes Metzger et al. $^{4}$

The gold-standard for medical treatment of gestational diabetes is insulin Singh et al. ${ }^{5}$ Although basal insulin is important, postprandial hyperglycemia is the major cause of macrosomia. Therefore, mealrelated bolus insulin is far more critical in treating women with gestational diabetes than is basal insulin. Rapid-acting insulin analogs are the treatment of choice for postprandial hyperglycemia in women with gestational diabetes. There are multiple reasons why most experts in gestational diabetes prefer insulin analogs over oral hypoglycemic agents; the most significant reason is that no oral agent will effectively treat postprandial hyperglycemia-the main driver of macrosomia. ${ }^{2}$

To date, only two rapid-acting insulin analogs have been shown to be safe and effective for the treatment of diabetes during pregnancy: insulin aspart and insulin lispro. ${ }^{6}$ Jovanovic and colleagues studied the metabolic and immunologic effects of insulin lispro in gestational diabetes by randomizing women to receive either regular human insulin or insulin lispro before consuming a test meal. ${ }^{7}$ The study proved the safety of insulin lispro for women with gestational diabetes. In a subsequent study, postprandial glycemic control was demonstrated in women with gestational diabetes taking insulin aspart via higher insulin peak and lower demand on endogenous insulin 
secretion. ${ }^{8}$ To date, there have not been any clinical trials evaluating the safety and efficacy of insulin glulisine in gestational diabetes.

The pharmacokinetics and pharmacodynamics of insulin glulisine are unique and insulin glulisine may be the best rapid-acting analog for the treatment of postprandial hyperglycemia. ${ }^{9,10}$ In this study we evaluated the safety and efficacy of insulin glulisine for the treatment of postprandial hyperglycemia in pregnant women with GDM. Our initial hypothesis was that insulin glulisine is non-inferior to currently proven rapid-acting insulin lispro when used in a basal/bolus regimen to treat hyperglycemia in patients with GDM.

\section{Methods}

\section{Subject recruitment site}

This study recruited pregnant women with GDM in Santa Barbara, CA, USA via the Santa Barbara County Public Health Department Diabetes in Pregnancy Clinic (SBCPHD-DPC). This clinic serves a population which is made up by Hispanic/Latina $(85 \%)$, white nonHispanic (10\%) and others (5\%). In this clinic, women diagnosed with gestational diabetes receive diet and exercise education.

\section{Inclusion and exclusion criteria}

We offered participation in this clinical trial to all women who met the following inclusion criteria: diagnosed gestational diabetes, history of failed lifestyle modification for treatment of GDM, pregnant and 20-32weeks gestation, and eat at least 2meals per day. Participants were excluded from the study based on the following: pregnant women $<18$ years old, blood pressure $>140 / 80 \mathrm{mmHg}$, $\mathrm{HbA} 1 \mathrm{c} \geq 6.5$ at time of enrollment, pre-pregnancy BMI $>40 \mathrm{~kg} / \mathrm{m}^{2}$, evidence of any fetal anomaly on any fetal ultrasound, currently using a hypoglycemic agent, refusal to use insulin before meals, inability to understand instructions or to consent to participate, history of type 1 or type 2 diabetes, or clinical judgment by investigator that patient is inappropriate for clinical trial or has a metabolic disorder that could interfere with results.

\section{Study procedure}

Within a single-center randomized controlled trial, we evaluated 16 women with GDM in two parallel arms. All women were on basal treatment with insulin NPH and were followed from enrollment to 38 weeks gestation. 6 women were randomized to the study arm (insulin glulisine) and 10 were in the control arm utilizing the current standard of care for meal-related bolus insulin (insulin lispro) and were started on insulin to treat their GDM using the dosing calculation shown in Table A. During weekly study visits self-reported blood and glucose diaries were collected and reviewed and insulin adjustments were made using the titration guideline shown in Figure A. In addition to weekly study visits, women participated in a four-hour in-clinic meal challenge which involved a standardized meal and blood glucose (YSI) measurements. ${ }^{11,12}$

The meal challenge was scheduled during weeks $2-6$ of treatment. Meal and rapid-acting insulin was standardized, as well as insulin NPH administration on the morning of the meal challenge. Morning NPH was administered at 0830 on day of the meal challenge by the investigator and was calculated based on weight and gestational age. Meal challenge commenced at 0900 with a time: -15 minute blood draw, standardized meal at 0915, with subsequent serum glucose YSI measurement every 15 minutes for 120 minutes, every 30 minutes for 60 minutes then every 60 minutes for one hour.
The primary efficacy measure for the study was glycemic control as measured by $\mathrm{HbAlc}$ from enrollment to 38 weeks gestation. Secondary efficacy measures include average postprandial glucose (PPG) throughout the study, the number of women with peak glucose at 75 minutes after the meal during the in-clinic meal challenge, incidence of macrosomia or large for gestational age (LGA) > $90^{\text {th }}$ percentile at delivery, incidence of primary cesarean section (excluding elective c-section), incidence of neonatal complications, and incidence of hypoglycemia during birth.

Starting insulin dose for subjects was calculated using Table 1 of the Appendix. Duration of treatment was from time of failed diet therapy to delivery (maximum of 22 weeks). Insulin was titrated weekly to maintain goal of pre-meal SMBG less than $90 \mathrm{mg} / \mathrm{dL}(5.0 \mathrm{mmol} / \mathrm{L})$ and postprandial SMBG less than $120 \mathrm{mg} / \mathrm{dL}(6.7 \mathrm{mmol} / \mathrm{L})$. NPH and rapid-acting analog were titrated simultaneously, to accommodate the constantly increasing insulin requirement which occurs during pregnancy. Titration was done according to the algorithm shown in Figure 1, found in the appendix. Furthermore, insulin was delivered subcutaneously in either the arm or thigh. Rapid-acting insulin analogs were provided via insulin delivery devices.

Table I Comparison of efficacy measures between intervention (glulisine) and control (lispro) groups

\begin{tabular}{|c|c|c|}
\hline Efficacy measure & $\begin{array}{l}\text { Intervention group, } \\
\mathrm{n}=10 \text { (Glulisine) }\end{array}$ & $\begin{array}{l}\text { Control group, } n=6 \\
\text { (Lispro)(Lispro) }\end{array}$ \\
\hline Avg. HbA Ic at enrollment & 6 & 5.7 \\
\hline $\begin{array}{l}\text { Avg. HbAlc throughout } \\
\text { study }(\mathrm{p}-\text { value }=0.06)\end{array}$ & 6 & 5.6 \\
\hline $\begin{array}{l}\text { Avg. PPG }(\mathrm{mg} / \mathrm{dL}) \\
\text { throughout the study } \\
(\mathrm{p} \text {-value }=0.5)\end{array}$ & 113 & 109 \\
\hline $\begin{array}{l}\text { Incidence of primary } \\
\text { cesarean sections }\end{array}$ & 0 & I \\
\hline $\begin{array}{l}\text { Incidence of neonatal } \\
\text { complications }\end{array}$ & 1 & 2 \\
\hline
\end{tabular}

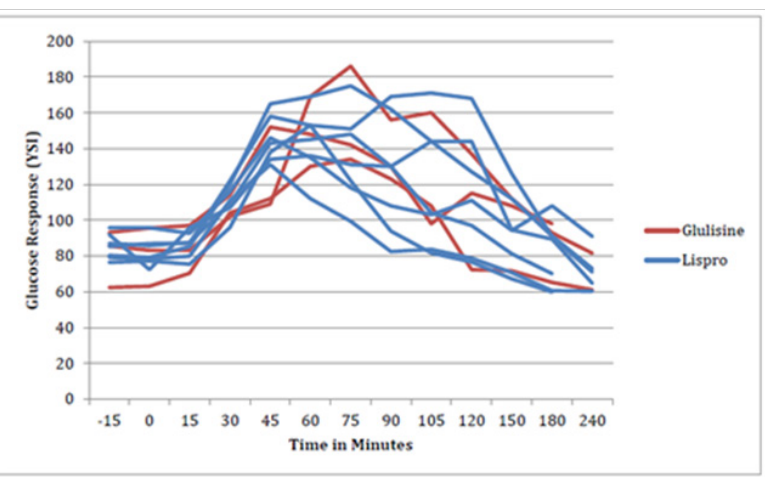

Figure I Participant glucose response during the meal challenge.

\section{Statistical analysis}

Statistical analysis was based on women who participated in the meal challenge and had more than 2 weekly visits ( 3 study arm women and 8 control arm women). Data analysis was done using SAS for Windows version 9. Differences between groups in continuous variables $(\mathrm{PPG}, \mathrm{HbAlc})$ were compared with t-tests and potential confounders (age, race/ethnicity) were controlled for with linear regression. Differences in categorical variables (neonatal complication events, macrosomia, and delivery type) were evaluated using chisquare or Fisher's tests as appropriate. We tested the hypothesis 
that the two treatments were different and the null hypothesis was equivalence. Non-inferiority was determined to be concluded if the upper limit of the $95 \%$ confidence interval around the mean difference in the postprandial glucose was less than the clinically meaningful difference of $10 \mathrm{mg} / \mathrm{dL} \cdot{ }^{13-16}$

\section{Results}

Of the 16 women enrolled in the study, three women dropped out of the study and one was lost to follow up. Within the study arm, two women dropped or were lost to follow up while two women dropped from the control arm. At enrollment, mean gestational age was 30.3 weeks with mean hemoglobin A1C (HbA1c) $6.0 \%$ in the study arm and 27.9 weeks with mean HbA1c 5.7\% in the control arm. Throughout the study, the average postprandial glucose (PPG) and $\mathrm{HbA} 1 \mathrm{c}$ measurements were $113 \mathrm{mg} / \mathrm{dL}$ and $6.0 \%$, respectively, for the study arm and $109 \mathrm{mg} / \mathrm{dL}$ and $5.6 \%$, respectively, for the control arm; these did not significantly differ between the two study groups ( $\mathrm{p}$-value $=0.5$ for $\mathrm{PPG}$ comparison and $\mathrm{p}$-value $=0.06$ for $\mathrm{HbA} 1 \mathrm{c}$ comparison; Table 1). Regarding the meal challenge, an equal number of women had peak blood glucose at 75 minutes after the meal $(n=2$ study arm and $\mathrm{n}=2$ control arm) as seen in Figure 1.

Secondary efficacy measures showed no statistically significant differences between study arm and control arm. No correlation was made between intervention and neonatal complications. There were two incidences of hypoglycemia reported during birth, one to the intervention arm and one to the control. Additionally, one notable incidence of jaundice was also reported in the control arm.

Of the remaining twelve women enrolled in the trial, there were no incidences of macrosomia or large for gestational age (LGA) > $90^{\text {th }}$ percentile at delivery in either arms of the study. One incidence of primary cesarean section (excluding elective c-section) was reported in the control arm, none in the study arm. Three repeat c-sections were reported incidentally to women who had a previous history of GDM. Four of the women reported induced vaginal deliveries. Two of the women had spontaneous vaginal deliveries. The two remaining women failed to report their delivery data.

\section{Conclusion}

Insulin glulisine is a rapidly acting analog with a pharmacokinetic and pharmacodynamic profile that is similar to both insulin lispro and insulin aspart. Insulin glulisine has a rapid onset, peak effect at $1 \mathrm{~h}$, and a shorter duration of action ( 4) when compared to human insulin. Clinical trials have demonstrated comparable or greater efficacy of insulin glulisine versus insulin lispro or regular insulin, respectively in the treatment of type 1 and type 2 diabetes Garg et al. ${ }^{17}$ To date, no clinical trials have been done to evaluate insulin glulisine for individuals with diabetes during pregnancy.

In this study results suggest that using insulin glulisine for the treatment of postprandial hyperglycemia in pregnant women with GDM may be safe and effective, but should be further evaluated. There was no significant difference between insulin glulisine and insulin lispro when evaluating the primary efficacy measure, average $\mathrm{HbA1c}$ for participants throughout the study. In addition, we found no differences in birth or neonatal complications, average PPG throughout the study, or time to reach peak glucose during the meal challenge. However, as this was a pilot study our sample size was not geared for statistical significance. Additional clinical trials are needed to confirm our results.

\section{Acknowledgements}

None.

\section{Funding}

Sanofi Investigator Initiated Study Program.

\section{Conflicts of interest}

The authors declare that they do not have conflicts of interest.

\section{References}

1. Centers for Disease Control and Prevention. National diabetes statistics report, 2017. Estimates of diabetes and its burden in the United States. GA: US Department of Health and Human Services, Centers for Disease Control and Prevention; 2017.

2. Castorino K, Jovanovič L. Pregnancy and diabetes management: advances and controversies. Clin Chem. 2011;57(2):221-230.

3. Johnson EL. Standards of Medical Care in Diabetes -2018/ ADA. Diabetes Care. 2018;41(Suppl 1):S13-S27.

4. Metzger B, Lowe L, Dyer A, et al. Hyperglycemia and adverse pregnancy outcomes. N Engl J Med. 2008;358(19):1991-2002.

5. Singh C, Jovanovič L. Insulin analogues in the treatment of diabetes in pregnancy. Obstet Gynecol Clin North Am. 2007;34(2):275-291.

6. Jovanovič L, Pettitt DJ. Treatment with insulin and its analogs in pregnancies complicated by diabetes. Diabetes Care. 2007;30 Suppl 2:S220-224.

7. Jovanovic-Peterson L, Peterson CM, Reed G, et al. Maternal postprandial blood glucose levels and infant birthweight: The Diabetes in Early Pregnancy Study. Am J Obstet Gynecol. 1991;164(1 Pt 1):103-111.

8. Jovanovic L, Pettitt DJ. Gestational diabetes mellitus. JAMA. 2001;286(20):2516-2518.

9. Becker RH, FrickAD. Clinical pharmacokinetics and pharmacodynamics of insulin glulisine. Clin Pharmacokinet. 2008;47(1):7-20.

10. Arnolds S, Rave K, Hovelmann U, et al. Insulin glulisine has a faster onset of action compared with insulin aspart in healthy volunteers. Exp Clin Endocrinol Diabetes. 2010;118(9):662-664.

11. Combs CA, Gunderson E, Kitzmiller JL, et al. Relationship of fetal macrosomia to maternal postprandial glucose control during pregnancy. Diabetes Care. 1992;15(10):1251-1257.

12. Manderson JG, Patterson CC, Hadden DR, et al. Preprandial versus postprandial blood glucose monitoring in type 1 diabetic pregnancy: a randomized controlled clinical trial. Am $J$ Obstet gynecol. 2003;189(2):507-512.

13. Jovanovic L, Druzin M, Peterson CM. The effect of euglycemia on the outcome of pregnancy in insulin-dependent diabetics as compared to normal controls. Am J Med. 1981;71(6):921-927.

14. Jovanovic L, Ilic, S, Pettitt D, et al. Metabolic and immunologic effects of insulin lispro in gestational diabetes. Diabetes Care. 1999;22(9):1422-1427.

15. Pettitt DJ, Ospina P, Kolaczynski JW, et al. Comparison of an insulin analog, insulin aspart, and regular human insulin with no insulin in gestational diabetes mellitus. Diabetes Care.2003;26(1):183-186.

16. Rhee MK, Ziemer DC, Kolm P, et al. Postchallenge glucose rises with increasing age even when glucose tolerance is normal. Diab Med. 2006;23(11):1174-1179.

17. Garg SK, Ellis SL, Ulrich H. Insulin glulisine: a new rapid-acting insulin analogue for the treatment of diabetes. Expert Opin Pharmacother. $2005 ; 6(4): 643-651$. 